\title{
The Clinical Outcome of Concurrent Speech Therapy and Transcranial Direct Current Stimulation in Dysarthria and Palilalia Following Traumatic Brain Injury: A Case Study
}

\author{
Masoumeh Bayat ${ }^{1,2}$, Malihe Sabeti ${ }^{3}$, K. S. Rao ${ }^{4}$, and Mohammad Nami ${ }^{1,4,5,6,7^{*}}$ \\ ${ }^{1}$ Department of Neuroscience, School of Advanced Medical Sciences and Technologies, Shiraz University of Medical \\ Sciences, Shiraz, Iran \\ ${ }^{2}$ Students' Research Committee, Shiraz University of Medical Sciences, Shiraz, Iran \\ ${ }^{3}$ Department of Computer Engineering, Islamic Azad University, North-Tehran Branch, Tehran, Iran \\ ${ }^{4}$ Neuroscience Center, Instituto de Investigaciones Científicas y Servicios de Alta Tecnología (INDICASAT AIP), City of \\ Knowledge, Panama City, Panama \\ ${ }^{5}$ DANA Brain Health Institute, Iranian Neuroscience Society, Fars Chapter, Shiraz, Iran \\ ${ }^{6}$ Department of Cognitive Neuroscience, Institute for Cognitive Science Studies-ICSS, Pardis, Tehran, Iran \\ ${ }^{7}$ Academy of Health, Senses Cultural Foundation, Sacramento, California, USA
}

\begin{abstract}
Introduction: Dysarthria, a neurological motor speech disorder, is regarded as a common sequala of traumatic brain injury (TBI). Palilalia is a speech disorder characterized by involuntary repetition of words, phrases, or sentences. Based on the evidence supporting the effectiveness of transcranial direct current stimulation (tDCS) in some speech disorders, we hypothesized that using tDCS would enhance the expected speech therapy outcome in a case of TBI with dysarthria and palilalia. Method: The "Be Clear" protocol, a relatively new approach in speech therapy in dysarthria, together with tDCS were employed in this single case investigation. With respect to the tDCS montage, regions of interest (ROIs) were identified based on the comparative analysis of resting-state vs. speech task-concurrent qEEG results. Results: Measures of intelligibility, an important index in the assessment of dysarthria, were superior to the primary protocol results immediately and 4 months after intervention. We did not find any factor other than the use of tDCS to justify this superiority. Palilalia showed a remarkable improvement immediately after intervention but fell somewhat after 4 months. This might have been justified owing to the subcortical origin of palilalia. Conclusion: Our present findings suggested that applying tDCS together with speech therapy might be more effective in similar case profiles as compared to traditional speech therapy. This notion needs to be systematically investigated in well-designed parallel arm clinical trials.
\end{abstract}

Keywords: traumatic brain injury (TBI); dysarthria; palilalia; transcranial direct current stimulation (tDCS); qEEG; speech therapy

Citation: Bayat, M., Sabeti, M., Rao, K. S., \& Nani, M. (2020). The clinical outcome of concurrent speech therapy and transcranial direct current stimulation in dysarthria and palilalia following traumatic brain injury: A case study. NeuroRegulation, 7(3), 118-128. https://doi.org/10.15540/nr.7.3.118

*Address correspondence to: Mohammad Nami, MD, PhD, Department of Neuroscience, School of Advanced Medical Sciences and Technologies, Shiraz University of Medical Sciences, Shiraz, Iran. Email: torabinami@sums.ac.ir

Copyright: (c) 2020. Bayat et al. This is an Open Access article distributed under the terms of the Creative Commons Attribution License (CC-BY).

\section{Edited by:}

Rex L. Cannon, PhD, SPESA Research Institute, Knoxville, Tennessee, USA

\section{Reviewed by:}

Rex L. Cannon, PhD, SPESA Research Institute, Knoxville, Tennessee, USA

Randall Lyle, PhD, Mount Mercy University, Cedar Rapids, Iowa, USA

\section{Introduction}

Traumatic brain injury (TBI) often results in significant neurofunctional deficits and/or evidence of brain pathology caused by an external force (Menon, Schwab, Wright, \& Maas, 2010). According to a local observational research (2007-2008), the incidence of TBI was estimated at 53.3-144 per 100,000 in Tehran (Rahimi-Movaghar, Saadat, Rasouli, Ghahramani, \& Eghbali, 2011). 
TBI potentially leaves patients with consequences in physical, sensory, cognitive, communicative, swallowing, and behavioral domains (ASHA, 2017). High-level cognitive functions, psychiatric disorders, and impairment of social and leisure activities are among long-term consequences of TBI (Stocchetti \& Zanier, 2016).

Along these lines, dysarthria is a communicative deficit regarded as one of the consequences of TBI. The condition is an acquired speech disorder which occurs following neurological injury of the motor component of the speech circuitry characterized by reducing the speech intelligibility due to poor, inaccurate, slow, or uncoordinated speech muscles (Mitchell, Bowen, Tyson, Butterfint, \& Conroy, 2017). This may possibly affect speech-related functions including respiratory, articulation, phonation, and resonance mechanisms (Kwon, Do, Park, Chang, \& Chun, 2015).

The mainstay of behavioral treatment in cases with stable dysarthria remains to be speech therapy which is yet a time-consuming procedure with relative outcomes (Mitchell et al., 2017). Speech therapy may focus on enhancement of particular speech subsystem through strengthening orobuccal musculature, implementing behavioral changes such as decreasing speaking rate and accurate pronunciation of speech phonemes by focusing on the kinetic, kinematic, and somatosensory aspects of speech production to improve intelligibility (Robertson, 2001; Yorkston, Hakel, Beukelman, \& Fager, 2007) or providing assistive devices to enhance communicative interactions (Yakcoub, Selouani, \& O'Shaughnessy, 2008).

Researchers have recently developed a program named "Be Clear" comprising a treatment plan based on Principles of Motor Learning (PML) which is a relatively new approach in treating motor disorders and believed to facilitate retention and transfer of skilled movement (Maas et al., 2008). The program is typically scheduled for 16 one-hour sessions and 15 minutes of homework over 4 weeks. Unlike traditional approaches, the program is based on external attentional focus (instead of internal attentional focus), intensive treatment, and practice schedule emphasizing on meaningful speech production tasks (Park, Theodoros, Finch, \& Cardell, 2016). Park et al. examined cases with dysarthria and deteriorating sentence intelligibility following TBI. In their prospective evaluation, they considered decreasing in speech rate as one of the most relevant correlates of treatment outcome just immediately and 3 months after the intervention
(Park et al., 2016). Meanwhile, no significant improvement was observed in terms of word intelligibility and psychosocial impact of dysarthria from the perspective of the speaker (Park et al., 2016).

Although the incidence of dysarthria following TBI is estimated at almost $60 \%$ (Mitchell et al., 2017), to date, there are few investigations on neurorehabilitation approaches using the concurrent use of electrical or magnetic brain stimulation and speech therapy. To our best knowledge, the effectiveness of such techniques in TBI-induced dysarthria has similarly not been articulated. As such, further research is required to examine the effectiveness of these approaches associated with common behavioral treatments given the high incidence of TBI as well as sever communicative problems in TBI patients who suffer from dysarthria.

Palilalia is a type of motor perseveration involving speech, consisting of compulsive repetition of normally articulated phrases, words, or syllables often with increasing rapidity and decreasing volume. Palilalia has been described in several neurological disorders such as cerebrovascular and degenerative diseases, encephalitis or tic disorders (Landi et al., 2012). Basal ganglia involvement has been suggested as the culprit in some cases of palilalia. Palilalia can be seen in untreated schizophrenic patients, in paramedian thalamic damage, also in advanced stages of degenerative brain diseases such as Alzheimer's disease, and in cerebrovascular or traumatic lesions of the basal ganglia (Azevedo et al., 2012; Van Borsel, Bontinck, Coryn, Paemeleire, \& Vandemaele, 2007) which the latter case is likely to be about our case.

Transcranial direct current stimulation (tDCS) is a noninvasive procedure in which brain cortices get potentiated for depolarization by an electrical field with a maximum 2 milliampere $(\mathrm{mA})$ direct current and electrodes localized on definite area over the scalp. The effectiveness of using tDCS in chronic motor disorders (Chang, Choi, \& Tseng, 2017), dysarthria (You, Chun, Kim, Han, \& Jung, 2010), and language impairments (Devido-Santos et al., 2013) due to stroke has been substantiated through brain imaging (Stagg \& Johansen-Berg, 2013). In various conditions including memory problems, executive dysfunctions, as well as issues with cognitive agility in chronic and subacute conditions in TBI, tDCS has been successfully applied (Demirtas-Tatlidede, Vahabzadeh-Hagh, Bernabeu, Tormos, \& Pascual-Leone, 2012). According to a systematic review, common protocols of tDCS have 
not associated with serious and irreversible side effects across over 33,200 sessions in 1000 subjects who underwent repeated sessions (Bikson et al., 2016).

The montage of tDCS electrodes is based on related study findings or neuroimaging techniques such as functional magnetic resonance imaging (fMRI), positron emission tomography (PET), or electroencephalography (EEG). EEG is a method to record and measure brain's electrical activity. Electrical brain signals or electroencephalogram contained regular patterns that may be better understood by their common spatial patterns (i.e., frequency range and amplitude). Bursts of sinusoidal waves occurred and reoccurred in a predictable fashion are corresponded with mental states. Indeed, today's advances in computer science and artificial intelligence have paved the way for new and faster analytical methods in digitally recorded signals, determining specific patterns in signals, and saving the digital data. Quantitative EEG (qEEG) is then a powerful and sensitive tool for identifying maladaptive brain activity patterns (Kaiser, 2007).

Based on the above, we hypothesized that a qEEGinformed tDCS intervention could potentially enhance the effectiveness of speech therapy in a client with chronic dysarthria following TBI. To test the hypothesis at least in a single case investigation, we applied the constructs of the Be Clear protocol in dysarthria together with tDCS. As such, an individualized therapy plan was formulated in Persian and applied to the patient. Because of the strong relationship between information transfer and speech intelligibility in dysarthria (Beukelman \& Yorkston, 1979), this measure was applied as a primary index in speech assessment. In summary, the findings of the current study revealed tES concurrent with speech therapy could yield more effectiveness compared to the standard practice of speech therapy in cases with TBI. This may then be regarded as a promising treatment plan in TBIrelated language problems in the future. An overview of diagnostic and therapeutic procedures is illustrated in Diagram 1.

Referral as affected by chronic dysarthria duo to TBI by SLT

Interview \& assessment

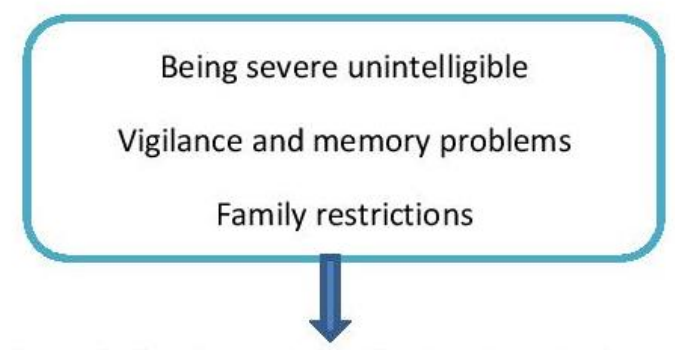

Designing an intensive and effective treatment plan

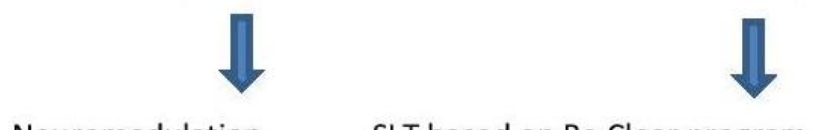

Neuromodulation $\quad$ SLT based on Be Clear program

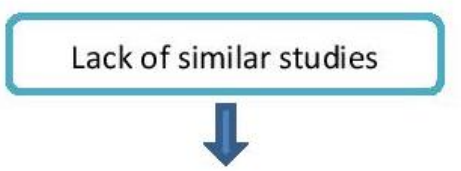

Detecting of ROI according to QEEG

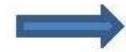

tDCS

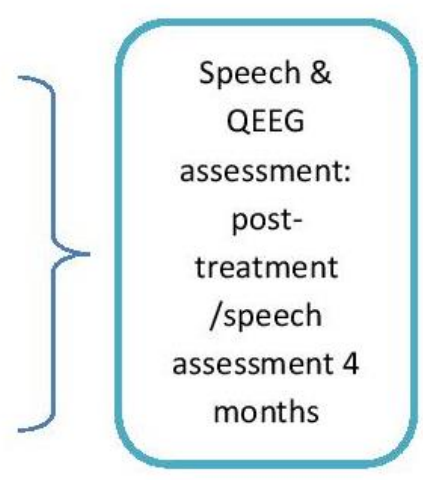

Diagram 1. Flow chart of the study. 


\section{Methods}

\section{Case Presentation}

MA was a 40-year-old male who had a car crash 5 years prior to presentation and experienced a closed head injury resulting in hospitalization, whereby he survived 31 days in a coma during his ICU admission. The patient had a master's degree in geology and used to work in a state-owned company prior to the accident. $\mathrm{He}$ was diagnosed with diffused axonal injury in his course of admission. The case had lost his orientation, movement, speech, and efficient swallowing for 6 months after which started to gradually regain some functions following intensive rehabilitation. The case was referred as dysarthria following TBI because of no progress through traditional speech therapy over last 3 years by his speech language therapist.

Informed consent was obtained for each experiment. All procedures related to the present investigation were approved by the Ethics Committee of Shiraz University of Medical Sciences (IR.SUMS.REC.1397.799).

Following the initial assessments by a clinical neuroscientist and speech language pathologist, he was found to have mild left-sided hemiparesis, left mild facial paresis and oral apraxia, decreased frequency and amplitude of oral movements, hypoesthesia in left upper extremity, slight gait paresis, and left upper extremity hyperreflexia. There was no hearing problem in his history as well as our observation.

Speech problems. The patient had significant communication problem where unintelligibility was the chief complaint. The family was deeply concerned about his excessive repetition of his own words and sentences in conversations.

Cognitive problems. He was found to have notable problem in short-term and episodic memory upon cognitive profile that had potentially caused difficulty in his social functions. Apparently, he was unable to recall what the breakfast was or how he came to our Brain Laboratory on the day of initial assessment.

\section{Diagnostic Assessments and Interventions}

Speech and language assessments. According to our speech language pathologist (SLP), the case was diagnosed with spastic dysarthria. Speech intelligibility index, diadochokinetic rate, maximum phonation time, speech rate, and the percentage of repeated words in all words were calculated. Strained and struggled phonation in open vowels and back consonants were diagnosed upon perceptual analyses.

For the assessment of intelligibility, as per the $\mathrm{Be}$ Clear protocol, eight conversational speech samples (approximately 40 seconds), presented in four paired comparisons, on topics of the participant's professional interests were given. The outcome was rated by four native Persian listeners in terms of clarity or understandability. The speech samples were randomly presented to listeners in several different combinations including pretreatment/posttreatment, (2) pretreatment/followup, (3) posttreatment/pretreatment, and (4) followup/pretreatment. The listeners' task was to determine whether the first or the second sample of each pair was easier to understand, or whether there was no discernible difference. Listeners were blinded to the assessment intervals (i.e., pretreatment, posttreatment, follow-up) and had no confrontation with the dysarthric speech. They were 25-45 years old and have undergraduate degrees (their email address is available for further correspondence). Prior to task completion, the listeners were provided with the following instructions adopted from the Be Clear protocol (Park et al., 2016):

You are going to hear pairs of audio speech samples. You will be deciding which speech sample, the first or the second, is clearer or easier to understand. On your paper you will write the name of a sample is easier to understand. If you do not think there is any difference in how easy it is to understand the two samples, write the word same. Repeat this procedure after one week and date it. There is a training sample at the first to listen and judge (Park et al., 2016).

Each listener completed the ratings twice with a 1-week interval. A total of 32 ratings comparing the pretreatment and posttreatment/follow-up speech samples were also included in the analysis.

Speech intelligibility is of basic considerations in dysarthria intervention (Hustad, 2006) and some objective methods have been suggested for its measurement. Of basic objective measurement methods is transcription the words of speakers' sentences by the listeners and then dividing the words correctly discriminated by whole words. The percentage of intelligibility is obtained by multiplying the result by 100 (Miller, 2013). Formal assessment of intelligibility was accomplished with Assessment of Intelligibility of Dysarthric Speech (ASSIDS) 
(Yorkston, Beukelman, \& Traynor, 1984) in the Be Clear program. The subjects are required to repeat a list of words and sentences after examiner and percentage of intelligibility is estimated in ASSIDS by transcription of speakers' responses. There is no such reliable test in Persian, so six samples of paired comparisons, two samples from each stage, were randomly chosen and transcribed by two independent SLPs. Then, the SLP calculated the percentage of words which were correctly transcribed. The final estimation was confirmed by the independent SLPs. In addition, the rate of speech was extracted in the same way. As the sample extraction method had to be consistent throughout the investigation (Miller, 2013), the content of participant's monologues which were about his professional major (geology) were recorded in the presence of a listener and the project SLP by a voice recorder Android software 1 $\mathrm{m}$ from his mouth and samples with 30-46 seconds connected speech with a coherent topic were selected and delivered to independent listeners for perceptual analyses and objective measurements.

The set of speech assessments adopted from the Be Clear protocol was carried out just before and after interventions as well as 4 months after interventions to assess dysarthria. The index of repeated words was applied to assess palilalia. The percentage of repeated words in whole words characterizing palilalia was calculated in speech samples by the main SLP based on independent SLTs' transcriptions.

Diadochokinetic rate (DDR) and maximum phonation time (MPT) were evaluated by independent SLPs besides the items stated in main protocol since these two aspects are affected in dysarthria (Kwon et al., 2015; Mitchell et al., 2017; Portnoy \& Aronson, 1982). DDR, which is also known as alternative motion rate, is an index used in clinical neurology and speech and language pathology to assess orofacial function and speech motor control and could be an indicator for rehabilitation efficacy (Yang, Chung, Chi, Chen, \& Wang, 2011). The rate is estimated by repetition of some nonsense syllables (/pe/, /te/, /ke/, each one 20 times, and /pe,te,ke/ 10 times) as fast as possible and dividing the number by the times which is known as Fletcher test (Fletcher, 1972).

Respiration is another affected aspect in dysarthria since reduction or alteration in respiratory support influences the airstream needed for phonation and articulation (Speyer et al., 2010). The maximum phonation time is proven to be a noninvasive, economical, and highly reliable evaluation in voice assessment and provides an objective measure indicating respiratory system efficacy through phonation (Speyer et al., 2010). Subjects are required to sustain a vowel after a deep inhalation for as long as possible at a comfortable pitch and loudness on one exhalation, without straining in this evaluation. The recorded samples during three stages of the study were submitted to independent SLPs to record the time in seconds up to two decimal places. Three subsequent trials were averaged to yield an estimation.

Aphasia was ruled-out based on Persian Aphasia Battery (PAB) developed by Nilipour (Nilipour, Pour Shahbaz, Ghoreishi, \& Yousefi, 2016). There were no resonance problems according to our SLP.

Electrophysiological assessments and tDCS application. Since there was no research on the use of tDCS in dysarthria following TBI, regions of interest (ROIs) were identified based on deviant brain electrophysiological patterns in speech tasks and resting-state qEEG compared with normal expected patterns. The EEG data was recorded from 19-channel NRsign amplifier according to the international 10-20 system. The impedance in the plug-in was set to a maximum of $5 \mathrm{~K} \Omega$. Data acquisition was performed at a frequency of at least 0.5 and a maximum of $40 \mathrm{~Hz}$, and the sensitivity was adjusted to $70 \mathrm{~Hz}$. An in-built NrSign and NeuroGuide software packages (NrSign, BC, Canada, 2011, and Applied Neuroscience Inc., 2017, respectively) were employed for data analysis.

EEG data was recorded in two conditions-upon resting eyes-open state and while performing speech tasks (reading, monologue, and orofacial movements). A minimum of 5 minutes of continuous signal was recorded. Signals were recorded while the participant was sitting on a comfortable chair.

Based on the discretion of two clinical neuroscientists, the therapy was planned using an individualized dual-channel montage for tES. A 2-mA anodal current was applied on $\mathrm{F} 7$ and $\mathrm{T} 5$ areas (based on the 10-20 system), while the cathod electrodes were placed over F6 and T4 areas during speech therapy sessions (Figure 1). A calibrated DC-stimulator delivered tDCS (Neurostim2, Medina Teb Ltd, Tehran). The electrode pads $(35 \times 35 \mathrm{~mm})$ were covered by equisized sponges soaked with $0.9 \%$ saline solution. 19-channel qEEG signals were acquired and analyzed upon speech tasks immediately after intervention. Results have been illustrated in Figure 2. 


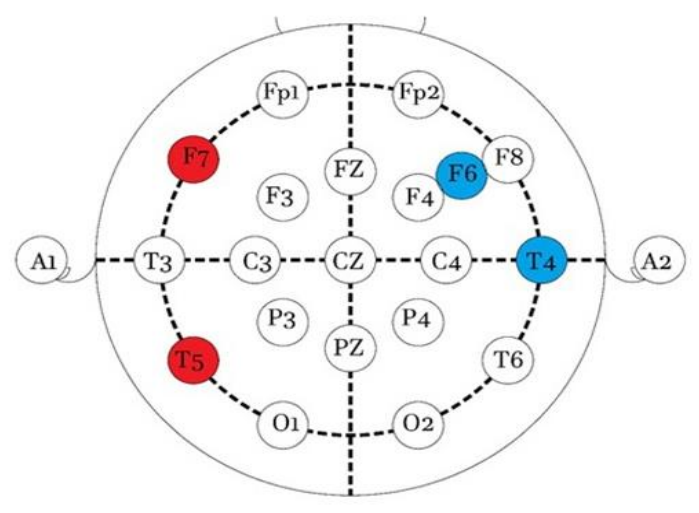

Figure 1. Regions of interest for tDCS. Red: Anodal tDCS, Blue: Cathodal tDCS.

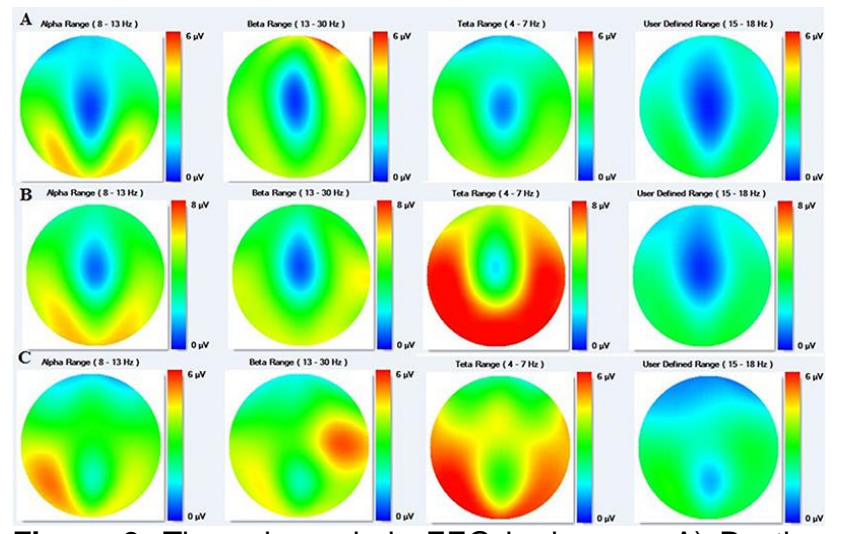

Figure 2. The color-coded qEEG brain map. A) Resting state: focal slowing at P3, P4, T8, T6, and C2 favoring nonspecific brain dysfunction, B) upon speech tasks preintervention: high amplitude for theta frequency in bilateral centrotemporal as well as bioccipital brain regions, C) upon speech tasks postintervention: increasing power of beta range frequency in right centrotemporal.

Behavioral intervention based on $\mathrm{Be}$ Clear protocol. The plan of speech therapy was developed in Persian based on the $\mathrm{Be}$ Clear protocol with regard to the participant's needs and performed in 10 consecutive 45 -min sessions over a 2-week period (5 days a week at 11:00 am) concurrently with tDCS. Since the treatment design was not language dependent, we translated the instructions of the protocol and customized it based on our participant's issues while clinging to instructions. The treatment comprised two phases including a prepractice and an intensive practice phase. All treatment sessions were delivered by the SLP in a one-to-one setting. The 1-hour prepractice phase aimed to establish the subject's understanding about the concept of clear speech production. Speech models were produced in two forms of normal and exaggerated more intelligible articulation by the SLP. The participant was asked to identify which of the speech samples were clearest and then discuss the changes made by the SLP (e.g., speech rate reduction, exaggerated articulation, and no repetition of words and phrases) which might have reflected in the observed improvements in speech clarity.

The knowledge of performance (KP) feedback on the client's speaking technique (e.g., speaking with open mouth, controlling the speech rate using fingers, and soft contact of vocal folds to reduce strained/struggled voice due to dysarthria) was provided in this session to shape a clearer speech. Clear speech refers to a speaking style where individuals spontaneously modify their habitual speech to enhance intelligibility to the listener (Park et al., 2016). The intensive practice phase followed the prepractice phase and consisted of 45-min therapy sessions, five times a week, for 2 weeks. Every session of this phase was initialized with providing an appropriate model and KP feedback by the SLP to shape proper speech through structured speech drills once he was able to produce adequate clarity (Ludlow et al., 2008).

Later during the sessions and consistent with the original protocol, the Be Clear program, reading, picture description, and conversation were delivered in small blocks of trials since PML-based small blocks of trials were expected to result in superior retention and transfer of trained skills than either traditional blocked or random practice schedules (Park et al., 2016).

During the intensive practice sessions, treatment stimuli were created on the basis of participant's interests and functional needs. The specific practice of meaningful speech production tasks was ensured to conform with the principles of specificity and saliency, potentially enhancing the effects of treatment on neuroplasticity (Wulf, 2013). Complete clarity during performing all tasks was ensured by encouraging the participant to focus on his acoustic speech features. The participant was asked to evaluate his speech to improve self-evaluation skills. During the practice phase of each session, the clinician provided general knowledge of results $(K R)$ feedback on speech clarity, labeling speech attempts as either clear or unclear since according to PML an external attentional focus (attentional focus on the external signals following a movement) promotes automaticity, retention, and transfer the outcomes (Hustad, Dardis, \& Mccourt, 2007). According to the Be Clear protocol, the latter part of 
the intensive phase focused on fulfilling homework which was not accomplished because of the mentioned problems.

A part of the present investigation was designed to assess the participant's palilalia given the family concerns. Due to the rare occurrence of such a speech disorder, many specific characteristics of palilalia are yet unknown (Akbari \& Shollenbarger, 2016). Therefore, we planned an intervention program based on PML principles. Given the SLP's diagnosis of palilalia in the present case, in intensive practice phase, the participant was required to provide feedback based on his acoustic speech features (based on external attentional focus rule in $\mathrm{PML}$ ) if there were repetitions. Then he had to discriminate the words which had been repeated and number of repetitions in upon his practice of productive speech.

\section{Results}

\section{Speech Analyses}

Results of the perceptual analyses of speech intelligibility were compared with normative outcome (Table 1) as per the Be Clear program, developed by Park and colleagues (Park et al., 2016).

\section{Table 1}

Results of comparative ratings for speech intelligibility.

\begin{tabular}{lcccc}
\hline & Pre better (\%) & Post better (\%) & FU better (\%) & Same (\%) \\
\hline Current study & 0 & 46.875 & 46.875 & 6.0 \\
Original study & 14.6 & 36.500 & 33.300 & 15.6 \\
\hline
\end{tabular}

Note. FU corresponds to samples of follow-up assessment, PR to samples of pretreatment assessment, and PT to samples of immediately posttreatment assessment. Pre: pretreatment; post: posttreatment; FU: follow-up.

The paired comparison ratings of speech intelligibility reported from the Be Clear program, for better illustration, was averaged for six participants who experienced dysarthria following TBI and presented in terms of percentage. Posttreatment and follow-up speech samples were rated better than pretreatment, $10 \%$ and $13 \%$ more than the $\mathrm{Be}$ Clear program, respectively.

The posttreatment and follow-up intelligibility gain in the Be Clear program was 8.36 and 6.99, whereas they were 38.3 and 24.7 in our study, respectively. Intraclass correlation coefficient (ICC) estimate and its $95 \%$ confident intervals was calculated using SPSS package (IBM SPSS statistics 22) based on the absolute-agreement, 2-way random-effects model. The ICC of two raters was $0.967(\mathrm{Cl}: 0.801-$ $0.995, \alpha=0.05$ ) which was considered as significant. Results have been illustrated in Table 2 for pretreatment, posttreatment, and follow-up phases.

\section{Table 2}

Results for sentence intelligibility in comparison with the original study.

\begin{tabular}{clccc}
\hline & & Pre & Post & FU \\
& & $M(S D)$ & $M(S D)$ & $M(S D)$ \\
\hline $\begin{array}{c}\text { \% Sentence } \\
\text { Intelligibility }\end{array}$ & This Study & 53.53 & 91.82 & $\mathbf{7 8 . 1 9}$ \\
& Original Study & $86.55(16.39)$ & $94.91(7.31)$ & $\mathbf{9 3 . 5 4 ( 1 1 . 0 3 )}$
\end{tabular}

Note. Pre: pretreatment; Post: posttreatment 1; FU: follow-up. 
Results of DDR and MPT for pretreatment, posttreatment, and 4 months after treatment are summarized in Table 3 . The only measures which increased with the intervention and remain better than initial assessment were $/ k e /$ and MPT that improved by $1 \mathrm{~s}$.
The percentage of repeated in whole words decreased by $16.17 \%$ in posttreatment assessment, yet it increased by $11.42 \%$ after 4 months (see Table 4). This has however remained more than $4 \%$ better than the initial assessment.

\section{Table 3}

Comparative outcomes for diadochokinetic rate and maximum phonation time.

\begin{tabular}{ccccc}
\hline & & Pre & Post & FU \\
& /pe/ & 3.6 & 3.6 & $\mathbf{2 . 5 3}$ \\
Diado & te/l & 2.2 & 1.9 & $\mathbf{1 . 7 0}$ \\
& /ke/ & 1.2 & 1.9 & $\mathbf{2 . 3 5}$ \\
\hline MPT & /pe, te, ke/ & 1.2 & 1.7 & $\mathbf{1 . 4 0}$ \\
\hline
\end{tabular}

Note. Pre: pretreatment; Post: posttreatment; FU: follow-up; MPT: maximum phonation time.

\section{Table 4}

Mean of speech rate and percentage of repeated words.

\begin{tabular}{cccc}
\hline & Pre & Post & FU \\
\hline WPM & 75.78 & 60.78 & $\mathbf{5 7 . 0 0}$ \\
PRW \% & 25.58 & 9.41 & $\mathbf{2 0 . 8 3}$ \\
\hline
\end{tabular}

Note. WPM: words per minute; PRW: percentage of repeated words.

\section{Everyday Communication Outcomes}

The psychological impact of dysarthria from the perspective of speaker was investigated with Dysarthria Impact Profile (DIP), a questionnaire in five sections, over three assessments phases within the Be Clear program (Walshe, Peach, \& Miller, 2009). DIP evaluates the impacts of dysarthria on the affective and communicative aspects. Since the questionnaire had not been translated into Persian, the participant was asked to describe his communicative alterations. He wrote:

Previously, almost no one could understand my words, but I trust I'm doing much better now. My memory has picked up. In addition, my relationship with friends has notably improved. I could recently go for field visits to evaluate two mines as a part of my job responsibilities and could more confidently provide a verbal report in our meetings. I am going to the office once or twice a week. I am kind of confident to get rehired.
Since this study focused solely on speech skills, the participant's cognitive profile was not thoroughly measured; meanwhile, his subjective as well as family reports appeared to indicate a notable progress.

\section{qEEG Measures}

Resting-state 19-channel EEG data suggested a focal transient slowing at P3, P4, T8, T6, and C2 derivations favoring nonspecific brain dysfunction. Spectral topography suggested an increased alpha amplitude in posterior brain regions.

The NRsign software was used to analyze the realtime data with respect to spectral and spatial distribution of the brain waves including $\alpha$ (alpha), $\beta$ (beta), $\theta$ (theta) frequency, and $\beta 2$ as the user defined range $(15-18 \mathrm{~Hz})$.

Accordingly, the task-concurrent data analyses revealed an event-related desynchronization (ERD) at T4 and T3 with episodic slowing at T5, T6, P3, and P4. The ERD was also seen in FP2, F4, and F3 upon task-concurrent EEG. Based on the analysis, 
the bihemispheric frontal polar areas and frontotemporal $\beta 2(15-18 \mathrm{~Hz})$ were planned to gain through tES neuromodulation.

General assessment of the EEG signals showed no abnormality in terms of focal or paroxysmal EEG signals including sharp waves or spikes across brain regions.

The amplitude of distributed signals was mapped on a multiwindow color-coded (heat) maps showing an increased amplitude for $\theta$ frequency in bilateral centrotemporal area as well as bioccipital brain regions. In the right centrotemporal, $\beta$ power was increased when the patient was reassessed following interventions, after 10 sessions as outlined in Figure 2-C. The subsequent qEEG-based evaluation demonstrated an improved spectral and spatial distributions in brain waves showing that $\theta$ and $\alpha$ distributions were more pronounced in specific brain regions predominantly involved in speech. There was also an apparent $a-\theta$ coherence in the left posterior brain regions as well as centrotemporal areas. The spectral up-band was set at $6 \mu \mathrm{V}$ across frequency bands.

\section{Discussion}

In this study, we investigated the feasibility of an intensive treatment, adopted from the Be Clear program combined with tDCS-based neuromodulation, to improve speech intelligibility in a patient with dysarthria following TBI.

Our patient demonstrated remarkable improvement following intervention in terms of perceptual ratings of speech intelligibility which sustained upon a 4month follow-up. Based on our findings, we could attribute the superiority in outcome to the application of tDCS in speech and language disorders (Baker, Rorden, \& Fridriksson, 2010; Marangolo, 2013; Monti et al., 2013). This might even be applicable for the improvement of language skills in healthy individuals (Sparing, Dafotakis, Meister, Thirugnanasambandam, \& Fink, 2008).

The striking issue with our participant was very low speech intelligibility at the prime of the process $(53.53 \%)$. However, this measure reached up to over $90 \%$ after the intervention. One of the negative findings in our investigation was an approximately $13 \%$ decrease in this measure after 4 months. Meanwhile, the intelligibility was still above $24 \%$ higher than the initial score. This can be at least partly justified by the short duration of the intervention.
Although the main study reported that the effect of intervention on improving communication attitudes was not significant, the participant in the present study presented a very positive report of his communicative progress. This can be attributed to very low initial intelligibility. Of course, we believe that the role of tDCS application should not be ignored. According to his report, it appears that such an improvement has partly been due to increased levels of the patient's conscious competence. This finding is consistent with studies investigated the effect of tDCS on the level of selfawareness in healthy individuals (Lauro et al., 2014) and patients with abnormal levels of consciousness (Bai et al., 2017; Zhang et al., 2017). The reduction in speech rate and decreased palilalia were other endpoints of this study. Although initial speech rate was lower than normal (about 75 words per minute), explosive pattern with fast words and phrases repetition following prolonged pauses due to spastic dysarthria and blocks in vowel and back consonants resulted in very low ineligibility along with an unusual pattern of speech. Memory and vigilance problems were thought to be contributing to these pauses. One of our clinical objectives was to reduce the speech rate to increase the patient's control over the acoustic speech signals using the KR feedback. The result of this intervention reflected in the reduction of repetitive words percentage in our posttreatment evaluation.

Despite the fact that the obtained clinical response in the reduction of speech rate remained sustainable, there has been an increase in the number of repetitions in the follow-up phase. Given the subcortical origins for palilalia, it seems that nonspecific, hypoxic brain damage in the present case has led to this predicament. Although tDCS and tACS have the potential to influence the abnormal cortical-subcortical networks which are involved in Parkinson's Disease (Hess, 2013), we suspect that the low efficiency of subcortical effects of tDCS resulted in an insufficient impact in the present case.

On the other hand, it seems that the application of KP feedback (easy onset) and KR feedback (attention to explosive nature of speech signal) resulted in an increased DDR of $/ \mathrm{ke} /$ in posttreatment and follow-up phases. Meanwhile, reduction of DDR of /pe/ and /te/ was contrary to our expectations. A plausible justification is that he tried to increase the clarity with a slowing down the rate of speech. 
In line with speech and vigilance alteration there were improvements in qEEG results. According to Gehrig, Wibral, Arnold, and Kell (2012), speech production tasks are expected to decrease a power primarily in visual and auditory cortices. A decrease in inhibitory a could engage these brain regions in the reading/speech production network; hence, $a$ decrease is markedly lateralized to the left and over the secondary auditory cortices (Gehrig et al., 2012). Moreover, the increased amplitude for $\theta$ frequency in bilateral centrotemporal areas as well as bioccipital brain regions which was found to be predominant in the left hemisphere in posttreatment and follows-up phases turned out to be consistent with our findings. On the other hand, though Giraud et al., believe that slow fluctuations in 3-6 Hz EEG rhythms most strongly correlate with the spontaneous neural activity over the right auditory region, higher-frequency fluctuations in the 28-40 $\mathrm{Hz}$ range shows left hemispheric predominance (Giraud et al., 2007). That said, we observed almost the opposite (i.e., an increased beta power over the right centrotemporal regions after the intervention).

The overall portrayal of the present case of TBIrelated chronic dysarthria shows a significant improvement in speech intelligibility (the most important symptom measured in dysarthria) after 2 weeks of intervention. This outcome, although at a single-case study level of evidence, may open new avenues to study such a tDCS-included protocol to ameliorate dysarthria symptoms following $\mathrm{TBI}$ in future sham-controlled clinical trials.

\section{Conclusion}

According to the present case's preliminary assessment and postintervention evaluation for speech function, we conclude that, although at a case-study level, tDCS may retain the potential in remediating speech insufficiencies, mainly intelligibility, in cases with TBI. Meanwhile, further research would be required to shed more lights on mechanistic peculiarities of such an approach in ameliorating speech predicaments following TBI. Further investigations to compare traditional speech therapy with sham- vs. true-tDCS would be warranted to explain the significance of such an approach in clinical settings.

\section{Author Disclosure}

Authors have no grants, financial interests, or conflicts to disclose.

\section{References}

Akbari, C., \& Shollenbarger, A. (2016). Acoustics of palilalia: A case study. Paper presented at the Proceedings of Meetings on Acoustics, 29(1), 172ASA. https://doi.org/10.1121 /2.0000605

American Speech-Language-Hearing Association (ASHA). (2017). Traumatic Brain Injury (TBI). Retrieved from http://www.asha.org/public/speech/disorders/TBI

Azevedo, J. C., Lopes, R., Curral, R., Esteves, M. F., Coelho, R., \& Roma-Torres, A. (2012). Clozapine-induced palilalia? Acta Neuropsychiatrica, 24(2), 122-124. https://doi.org/10.1111 /j.1601-5215.2011.00594.X

Bai, Y., Xia, X., Kang, J., Yang, Y., He, J., \& Li, X. (2017). TDCS modulates cortical excitability in patients with disorders of consciousness. Neurolmage: Clinical, 15, 702-709. https://doi.org/10.1016/j.nicl.2017.01.025

Baker, J. M., Rorden, C., \& Fridriksson, J. (2010). Using transcranial direct-current stimulation to treat stroke patients with aphasia. Stroke, 41(6), 1229-1236. https://doi.org /10.1161/STROKEAHA.109.576785

Beukelman, D. R., \& Yorkston, K. M. (1979). The relationship between information transfer and speech intelligibility of dysarthric speakers. Journal of Communication Disorders, 12(3), 189-196. https://doi.org/10.1016/0021-9924(79)900406

Bikson, M., Grossman, P., Thomas, C., Zannou, A. L., Jiang, J., Adnan, T., ... Woods, A. J. (2016). Safety of transcranial direct current stimulation: Evidence based update 2016. Brain Stimulation, 9(5), 641-661. https://doi.org/10.1016 /j.brs.2016.06.004

Chang, S.-H., Choi, J., \& Tseng, S.-C. (2017). Effects of tDCS on stepping reaction in healthy adults and individuals with chronic stroke. Brain Stimulation, 10(1), e4-e5. https://doi.org /10.1016/j.brs.2016.11.031

Demirtas-Tatlidede, A., Vahabzadeh-Hagh, A. M., Bernabeu, M., Tormos, J. M., \& Pascual-Leone, A. (2012). Noninvasive brain stimulation in traumatic brain injury. The Journal of Head Trauma Rehabilitation, 27(4), 274-292. https://doi.org /10.1097/HTR.0b013e318217df55

Devido-Santos, M., Gagliardi, R. J., Mac-Kay, A. P. M. G., Boggio, P. S., Lianza, R., \& Fregni, F. (2013). Transcranial direct-current stimulation induced in stroke patients with aphasia: A prospective experimental cohort study. Sao Paulo Medical Journal, 131(6), 422-426. https://doi.org/10.1590 /1516-3180.2013.1316595

Fletcher, S. G. (1972). Time-by-count measurement of diadochokinetic syllable rate. Journal of Speech and Hearing Research, 15(4), 763-770. https://doi.org/10.1044 /jshr.1504.763

Gehrig, J., Wibral, M., Arnold, C., \& Kell, C. A. (2012). Setting up the speech production network: How oscillations contribute to lateralized information routing. Frontiers in Psychology, 3(169). https://doi.org/10.3389/fpsyg.2012.00169

Giraud, A.-L., Kleinschmidt, A., Poeppel, D., Lund, T. E., Frackowiak, R. S. J., \& Laufs, H. (2007). Endogenous cortical rhythms determine cerebral specialization for speech perception and production. Neuron, 56(6), 1127-1134. https://doi.org/10.1016/j.neuron.2007.09.038

Hess, C. W. (2013). Modulation of cortical-subcortical networks in Parkinson's disease by applied field effects. Frontiers in Human Neuroscience, 7, 565. https://doi.org/10.3389 /fnhum.2013.00565

Hustad, K. C. (2006). Estimating the intelligibility of speakers with dysarthria. Folia Phoniatrica et Logopaedica, 58(3), 217-228. https://doi.org/10.1159/000091735

Hustad, K. C., Dardis, C. M., \& Mccourt, K. A. (2007). Effects of visual information on intelligibility of open and closed class words in predictable sentences produced by speakers with 
dysarthria. Clinical Linguistics \& Phonetics, 21(5), 353-367. https://doi.org/10.1080/02699200701259150

Kaiser, D. A. (2007). What is quantitative EEG? Journal of Neurotherapy, 10(4), 37-52. https://doi.org/10.1300 /J184v10n04_05

Kwon, Y. G., Do, K. H., Park, S. J., Chang, M. C., \& Chun, M. H. (2015). Effect of repetitive transcranial magnetic stimulation on patients with dysarthria after subacute stroke. Annals of Rehabilitation Medicine, 39(5), 793-799. https://doi.org /10.5535/arm.2015.39.5.793

Landi, D., Benvenga, A., Quattrocchi, C. C., Vollero, L., Assenza, G., Pellegrino, G., ... Tombini, M. (2012). Complex epileptic palilalia: A case report. Seizure: European Journal of Epilepsy, 21(8), 655-657. https://doi.org/10.1016 /j.seizure.2012.06.009

Lauro, L. J. R., Rosanova, M., Mattavelli, G., Convento, S., Pisoni, A., Opitz, A., ... Vallar, G. (2014). TDCS increases cortical excitability: Direct evidence from TMS-EEG. Cortex, 58, 99-111. https://doi.org/10.1016/j.cortex.2014.05.003

Ludlow, C. L., Hoit, J., Kent, R., Ramig, L. O., Shrivastav, R., Strand, E., ... Sapienza, C. M. (2008). Translating principles of neural plasticity into research on speech motor control recovery and rehabilitation. Journal of Speech, Language, and Hearing Research, 51(1), S240-S258. https://doi.org /10.1044/1092-4388(2008/019)

Maas, E., Robin, D. A., Hula, S. N. A., Freedman, S. E., Wulf, G., Ballard, K. J., \& Schmidt, R. A. (2008). Principles of motor learning in treatment of motor speech disorders. American Journal of Speech-Language Pathology, 17(3), 277-298. https://doi.org/10.1044/1058-0360(2008/025)

Marangolo, P. (2013). tDCS over the left inferior frontal cortex improves speech production in aphasia. Frontiers in Human Neuroscience, $\quad 7, \quad 539 . \quad$ https://doi.org/10.3389 /fnhum.2013.00539

Menon, D. K., Schwab, K., Wright, D. W., \& Maas, A. I. (2010). Position statement: Definition of traumatic brain injury. Archives of Physical Medicine and Rehabilitation, 91(11), 1637-1640. https://doi.org/10.1016/j.apmr.2010.05.017

Miller, N. (2013). Measuring up to speech intelligibility. International Journal of Language \& Communication Disorders, 48(6), 601-612. https://doi.org/10.1111/14606984.12061

Mitchell, C., Bowen, A., Tyson, S., Butterfint, Z., \& Conroy, P. (2017). Interventions for dysarthria due to stroke and other adult-acquired, non-progressive brain injury. Cochrane Database of Systematic Reviews. https://doi.org/10.1002 14651858.CD002088.pub3

Monti, A., Ferrucci, R., Fumagalli, M., Mameli, F., Cogiamanian, F., Ardolino, G., \& Priori, A. (2013). Transcranial direct current stimulation (tDCS) and language. Journal of Neurology, Neurosurgery, \& Psychiatry, 84(8), 832-842. http://doi.org/10.1136/jnnp-2012-302825

Nilipour, R., Pour Shahbaz, A., Ghoreishi, Z. S., \& Yousefi, A. (2016). Reliability and validity of Persian aphasia battery test. Iranian Journal of Ageing, 10(4), 182-191. Retrieved from http://salmandj.uswr.ac.ir/article-1-835-en.html

Park, S., Theodoros, D., Finch, E., \& Cardell, E. (2016). Be clear: A new intensive speech treatment for adults with nonprogressive dysarthria. American Journal of SpeechLanguage Pathology, 25(1), 97-110. https://doi.org/10.1044 12015_AJSLP-14-0113

Portnoy, R. A., \& Aronson, A. E. (1982). Diadochokinetic syllable rate and regularity in normal and in spastic and ataxic dysarthric subjects. Journal of Speech and Hearing Disorders, 47(3), 324-328. https://doi.org/10.1044 /jshd.4703.324

Rahimi-Movaghar, V., Saadat, S., Rasouli, M. R., Ghahramani, M., \& Eghbali, A. (2011). The incidence of traumatic brain injury in Tehran, Iran: A population based study. The American Surgeon, 77(6), E112-E114.
Robertson, B. D. (2001). Networked personal contact manager. U.S. Patent No. 6,269,369 B1. Washington, DC: U.S. Patent and Trademark Office.

Sparing, R., Dafotakis, M., Meister, I. G., Thirugnanasambandam, N., \& Fink, G. R. (2008). Enhancing language performance with non-invasive brain stimulation-A transcranial direct current stimulation study in healthy humans. Neuropsychologia, 46(1), 261-268. https://doi.org /10.1016/j.neuropsychologia.2007.07.009

Speyer, R., Bogaardt, H. C. A., Passos, V. L., Roodenburg, N. P. H. D., Zumach, A., Heijnen, M. A. M., ... Brunings, J. W. (2010). Maximum phonation time: Variability and reliability. Journal of Voice, 24(3), 281-284. https://doi.org/10.1016 /j.jvoice.2008.10.004

Stagg, C. J., \& Johansen-Berg, H. (2013). Studying the effects of transcranial direct-current stimulation in stroke recovery using magnetic resonance imaging. Frontiers in Human Neuroscience, $\quad 7, \quad 857 . \quad$ https://doi.org/10.3389 /fnhum.2013.00857

Stocchetti, N., \& Zanier, E. R. (2016). Chronic impact of traumatic brain injury on outcome and quality of life: A narrative review. Critical Care, 20(1), 148. https://doi.org /10.1186/s13054-0161318-1

Van Borsel, J., Bontinck, C., Coryn, M., Paemeleire, F., \& Vandemaele, P. (2007). Acoustic features of palilalia: A case study. Brain and Language, 101(1), 90-96. https://doi.org /10.1016/j.bandl.2006.06.118

Walshe, M., Peach, R. K., \& Miller, N. (2009). Dysarthria impact profile: Development of a scale to measure psychosocial effects. International Journal of Language \& Communication Disorders, 44(5), 693-715. https://doi.org/10.1080 113682820802317536

Wulf, G. (2013). Attentional focus and motor learning: A review of 15 years. International Review of Sport and Exercise Psychology, 6(1), 77-104. https://doi.org/10.1080 /1750984X.2012.723728

Yakcoub, M. S., Selouani, S.-A., \& O'Shaughnessy, D. (2008, March). Speech assistive technology to improve the interaction of dysarthric speakers with machines. Paper presented at the 2008 3rd International Symposium on Communications, Control and Signal Processing, St. Julians, Malta. https://doi.org/10.1109/ISCCSP.2008.4537398

Yang, C.-C., Chung, Y.-M., Chi, L.-Y., Chen, H.-H., \& Wang, Y.-T. (2011). Analysis of verbal diadochokinesis in normal speech using the diadochokinetic rate analysis program. Journal of Dental Sciences, 6(4), 221-226. https://doi.org/10.1016 /j.jds.2011.09.007

Yorkston, K., Beukelman, D., \& Traynor, C. (1984). Assessment of Intelligibility of Dysarthric Speech (KIT). Austin, TX: PROED, Inc.

Yorkston, K. M., Hakel, M., Beukelman, D. R., \& Fager, S. (2007). Evidence for effectiveness of treatment of loudness, rate, or prosody in dysarthria: A systematic review. Journal of Medical Speech-Language Pathology, 15(2), xi-xxxvi.

You, D. S., Chun, M. H., Kim, D. Y., Han, E. Y., \& Jung, S. E. (2010). The effects of transcranial direct current stimulation on dysarthria in stroke patients. Journal of Korean Academy of Rehabilitation Medicine, 34(1), 10-14.

Zhang, Y., Song, W., Du, J., Huo, S., Shan, G., \& Li, R. (2017). Transcranial direct current stimulation in patients with prolonged disorders of consciousness: Combined behavioral and event-related potential evidence. Frontiers in Neurology, 8, 620. https://doi.org/10.3389/fneur.2017.00620

Received: July 30, 2020

Accepted: September 16, 2020

Published: September 30, 2020 